\title{
Mapping strain fields in ultrathin bonded Si wafers by $x$-ray scattering
}

Nielsen, Mourits; Poulsen, Mette; Bunk, Oliver; Kumpf, Christian; Feidenhans'l, Robert Krarup; Johnson, R.L.; Jensen, Flemming; Grey, Francois

Published in:

Applied Physics Letters

Link to article, DOI:

$10.1063 / 1.1476702$

Publication date:

2002

Document Version

Publisher's PDF, also known as Version of record

Link back to DTU Orbit

Citation (APA):

Nielsen, M., Poulsen, M., Bunk, O., Kumpf, C., Feidenhans'l, R. K., Johnson, R. L., Jensen, F., \& Grey, F. (2002). Mapping strain fields in ultrathin bonded Si wafers by x-ray scattering. Applied Physics Letters, $80(18)$, 3412-3414. https://doi.org/10.1063/1.1476702

\section{General rights}

Copyright and moral rights for the publications made accessible in the public portal are retained by the authors and/or other copyright owners and it is a condition of accessing publications that users recognise and abide by the legal requirements associated with these rights.

- Users may download and print one copy of any publication from the public portal for the purpose of private study or research.

- You may not further distribute the material or use it for any profit-making activity or commercial gain

- You may freely distribute the URL identifying the publication in the public portal 


\title{
Mapping strain fields in ultrathin bonded Si wafers by x-ray scattering
}

\author{
M. Nielsen, ${ }^{\text {a) }}$ M. Poulsen, O. Bunk, C. Kumpf, ${ }^{\text {b) }}$ and R. Feidenhans'l \\ Risф National Laboratory, DK-4000 Roskilde, Denmark \\ R. L. Johnson \\ II. Institut für Experimentalphysik, Universität Hamburg, Luruper Chaussee 149, D-22761 Hamburg, \\ Germany \\ F. Jensen and F. Grey \\ Mikroelektronik Centret, Technical University of Denmark, DK-2800 Lyngby, Denmark
}

(Received 14 November 2001; accepted for publication 8 March 2002)

$\mathrm{X}$-ray scattering reveals the atomic displacements arising from rotational misalignment in ultrathin silicon bonded wafers. For a $4.3 \mathrm{~nm}$ top wafer, the strain field penetrates from the bonded interface to the surface and produces distinctive finite-size oscillations in x-ray data. Analytical calculations permit the atomic displacements throughout the thin top wafer to be modeled. (C) 2002 American Institute of Physics. [DOI: 10.1063/1.1476702]

When two $\mathrm{Si}(001)$ crystals are bonded directly with a small, well-defined, rotational misorientation, a strain field is created at the interface that may be characterized in terms of periodic lattices of dislocations. ${ }^{1}$ When the period between dislocations becomes larger than the thickness of the upper crystal then the strain field will penetrate to the top surface. ${ }^{2-5}$ Such bonded wafers have aroused considerable interest because they open up unique possibilities for device fabrication. ${ }^{6}$ Particularly interesting are applications that use them as templates in epitaxial growth ${ }^{7}$ or for creating nanometer periodic surface structures by selective etching. ${ }^{8}$

$\mathrm{X}$-ray scattering measurements of the strain field in thick bonded wafers with different twist angles $\theta_{\text {twist }}$ have been reported previously. ${ }^{1,9}$ These measurements were later extended to cover $\theta_{\text {twist }}$ from $0.2^{\circ}$ to $25^{\circ}$. We found that the thickness of the strained interface is about $0.8 \mathrm{~nm}$ for $\theta_{\text {twist }}$ $>8^{\circ}$ but for smaller angles the thickness is inversely proportional to $\theta_{\text {twist }}$, and for a twist angle of $0.2^{\circ}$, the thickness exceeds $30 \mathrm{~nm}$. In this regime we find that the strain amplitude decays exponentially from the bonded interface. In the present work we apply $\mathrm{x}$-ray scattering to measure the strain amplitudes in samples with an ultrathin bonded upper crystal and analyze the results using an isotropic elastic approximation. . $^{1,2,10,11}$

Silicon-on-insulator (SOI) wafers with a $200 \mathrm{~nm} \mathrm{Si(001)}$ crystal on top of the $\mathrm{SiO}_{2}$ were thinned by dry oxidation. The required layer thickness was determined using ellipsometry. The wafer was hydrophobically bonded to a standard Si wafer with a well-defined twist angle $\theta_{\text {twist }} \cdot{ }^{4}$ After annealing at $1000^{\circ} \mathrm{C},{ }^{9}$ the SOI bulk crystal and $\mathrm{SiO}_{2}$ layer were etched away leaving the thin top crystal bonded to the standard wafer.

The scattering measurements were performed with the $z$-axis diffractometer at the BW2 beamline in HASYLAB (Hamburg, Germany). The samples were mounted in a constant angle of incidence geometry. The $\mathrm{x}$-ray energy was 10

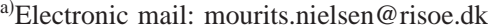

${ }^{b)}$ Present address: Experimentelle Physik II, Universität Würzburg, Am Hubland, D-97074 Würzburg, Germany.
}

$\mathrm{keV}$, the angular divergence of the incoming beam $<0.5 \mathrm{mrad}$, and the angular acceptance of the detector was set to $1 \mathrm{mrad}$ both horizontally and vertically. We present data for two samples with thicknesses of the upper crystal of 4.3 and $13.6 \mathrm{~nm}$, respectively. For both samples the twist angle was $2.1^{\circ}$ and the tilt angles were less than $0.3^{\circ}$. The measured intensities were corrected to account for the Lorenz factor, the effective diffracting volume of the sample, the width of the rocking curve full width at half maximum (FWHM), and integrated intensity calculated as peak intensity $\times$ FWHM.

The misfit creates a strain field characterized by a square lattice of screw dislocations in the bonding plane with a period of ${ }^{1,12}$

$$
\lambda=N_{x} a / \sqrt{2},
$$

where $a=0.543 \mathrm{~nm}$ is the cubic lattice parameter of $\mathrm{Si}$ and $N_{x}$ is given by

$$
N_{x}=1 /\left[2 \sin \left(\theta_{\text {twist }} / 2\right)\right] \text {. }
$$

We use surface coordinates with the (001) direction normal to the surface and (100) along the atomic rows in the surface plane. The dislocations are inclined by $\theta_{\text {twist }} / 2$ relative to the (100) directions of both crystals. For the firstharmonic component the 1/e length of the strain decay is equal to $\lambda / 2 \pi .{ }^{13}$ For samples with a thin upper crystal we include the effect of image forces in the model calculation of the displacements from the dislocations. ${ }^{3,10}$

The strain field produces satellite reflections $(S)$ in the plane parallel to the interface around the Bragg points $(P)$ of the two crystals. The satellite positions are determined by the reciprocal lattice of the square array of dislocations. The scattering intensity at satellite points is caused by the displacements from the bulk lattice sites in the two crystals and the strain is the derivative of the displacement field.

The fundamental wave vector $\mathbf{q}$ for the periodic displacement field is equal to the difference between the (101) Bragg points of the substrate crystal $P_{1}(101)$ and the upper crystal $P_{2}(101)$, shown in the inset to Fig. 2(c): 

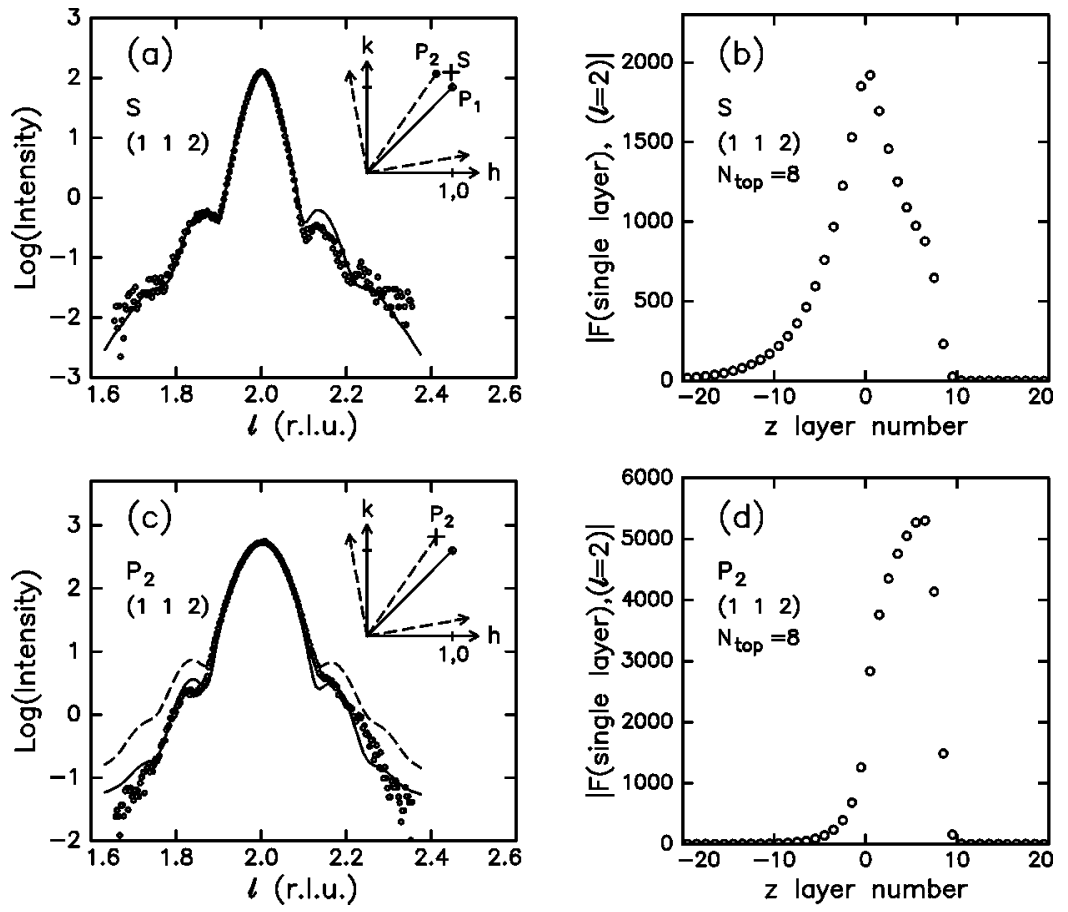

(3)

Satellites occur at $P_{1}(101)-\mathbf{q}$ and $P_{2}(101)+\mathbf{q}$, and also at $P_{1}(112) \pm \mathbf{q}$ and at $P_{2}(112) \pm \mathbf{q}$ and around all other bulk Bragg points.

The intensity profile measured in scans through satellite points in the direction perpendicular to the interface $(z$ direction in real space and $l$ direction in reciprocal space) reveals the changes in the displacements with increasing distance from the interface. ${ }^{1}$ For ultrathin upper crystals intensity oscillations are produced by the abrupt termination at the top surface.

Figure 1 shows $x$-ray scattering data from the sample with a $4.3 \mathrm{~nm}$ top crystal. Panel (a) shows an $l$ scan through a satellite near a (112) bulk Bragg reflection. The location of the satellite peak in the $(h k)$ plane at $l=2$ is indicated in the upper-right corner of Fig. 1; the reciprocal lattice axes of the bottom crystal are indicated by solid lines and those of the top crystal by dashed lines. At the satellite point $S(112)$ $=P_{1}(112)+\mathbf{q}$ marked by + in Fig. 1(a) the scattering intensity contains contributions from the first harmonic of the displacement field in both crystals so it is a relatively strong satellite.

The solid curve in Fig. 1 shows the calculated profile assuming homogeneous elastic properties. The best fit was obtained with a top surface roughness of $\sigma=0.6 \mathrm{~nm}$ and a model where the intensities from three different top crystal thicknesses, $N_{\text {top }} a=7 a, 8 a$ and $9 a$, were added. The mean slope in the wings of the curve determines the surface roughness. The surface-termination oscillations are damped by adding contributions from different top crystal thicknesses-in this case three were necessary. Panel 1(b) shows the structure-factor amplitude at $l=2$ of single atomic layers parallel to the interface for layers separated by $a$ $=0.543 \mathrm{~nm}$. If there were no surface termination at the top surface the function in Fig. 1(b) would be symmetric.

Figure 1(a) clearly demonstrates that the strain penetrates the entire upper crystal. Figure 1(c) shows a similar Downloaded 10 Mar 2010 to 192.38.67.112. Redistribution subject
FIG. 1. X-ray data and model calculations for a bonded wafer with a $4.3 \mathrm{~nm}$ top crystal. Panel (a) shows the integrated intensity in an $l$ scan through the satellite $S$ marked with + . The reciprocal lattice axes at $l=2$ of the substrate (solid lines) and top crystal (dashed lines) are indicated [the turn angle $\left(\theta_{\text {twist }}\right)$ being exaggerated for clarity]. The solid curve is the model calculation. Panel (b) shows the structure factor amplitude at $l=2$ for single atomic layers. Panels (c) and (d) show the corresponding data for an $l$ scan through the Bragg point $P_{2}\left(\begin{array}{lll}1 & 1 & 2\end{array}\right)$. scan from the same sample through the $P_{2}(112)$ Bragg point of the upper crystal together with the calculated profile. The best fit is obtained using the same parameters as in Fig. 1(a) except for a larger surface roughness of $\sigma=0.9 \mathrm{~nm}$. The general shapes of the two intensity curves is nicely reproduced by the model calculation.

Figure 2 shows similar results from the sample with a $13.6 \mathrm{~nm}$ top crystal. The intensity curves are calculated by summing contributions from crystal thicknesses $N_{\text {top }} a$ $=24 a, 25 a$, and $26 a$ with a surface roughness of $\sigma$ $=0.4 \mathrm{~nm}$. The same parameters, including the surface roughness, were used in Figs. 2(a) and 2(c). Here, we present results for the satellite point $P_{2}(101)+\mathbf{q}$ and the Bragg point $P_{2}$ (101). The asymmetry of the single-layer structure factor amplitude in panel (b) arises from the superposition of the first harmonic of the periodic displacement field in the upper crystal with the second harmonic in the substrate crystal. There are clearly no oscillations visible in Fig. 2(a), and the effect of the strain field at the surface is negligible. Figure 2(c) shows the distinct oscillations in the Bragg scattering intensity for the 13.6-nm-thick upper crystal.

The data presented here illustrate the wide range in realspace dimensions that can be realized for regular patterns of strained silicon. By establishing small misfit angles of a few tenths of a degree, the strain pattern parallel to the interface may have a $100 \mathrm{~nm}$ period and a perpendicular decay length of several $10 \mathrm{~nm}$. A bonded wafer with a $5 \mathrm{~nm}$ upper crystal will provide perfect matching with a zero substrate misfit incorporating an array of dislocations, each forming a dislocation pair together with its mirror dislocation above the surface. The displacements in the upper crystal will be dominated by the local field from the nearest dislocation and be anharmonic throughout the entire crystal. With larger misfit $\theta_{\text {twist }}$ or thicker upper crystal, when $N_{\text {top }} a>\lambda /(2 \pi)$, the displacement field becomes more harmonic towards the top surface and the displacement amplitude decreases exponentially. This is illustrated in Fig. 3, which shows the displacement AIP license or copyright; see http://apl.aip.org/apl/copyright.jsp 

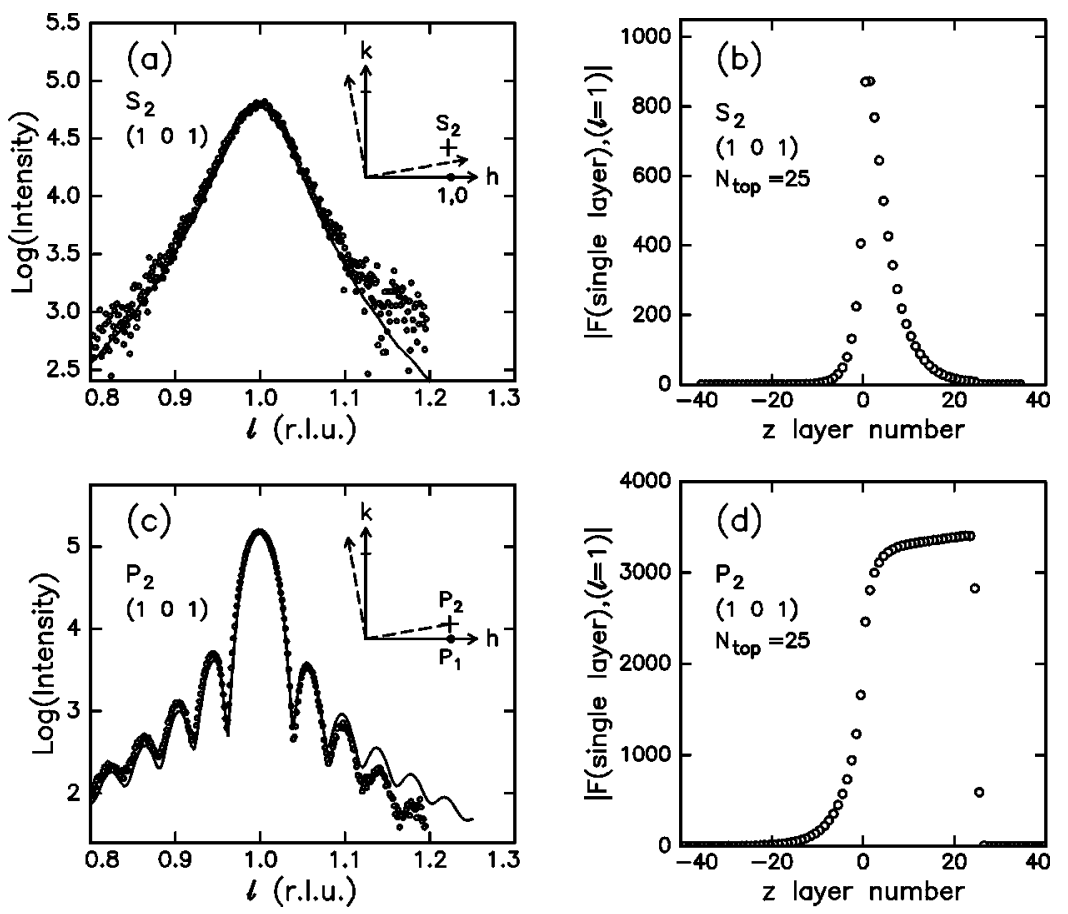

FIG. 2. X-ray data and model calculations for a bonded wafer with $13.6 \mathrm{~nm}$ top crystal. Symbols and curves are as in Fig. 1 but the inset in panel (a) shows the reciprocal lattice planes at $l=1$. function of the individual atomic layer parallel to the interface taken from the model calculation of Fig. 1(a). For clarity, the curves are vertically displaced by 0.25 , the interval between the atomic layers is $a$, and the units for both axes is $a / \sqrt{2}$. The arrow at $\lambda /(2 \pi)$ illustrates the borderline between the harmonic and the anharmonic regimes. In the latter, the almost linear slope regions of the curves correspond to almost matching nonrotated crystals. This sample has significant top surface strain, which will influence both the surface

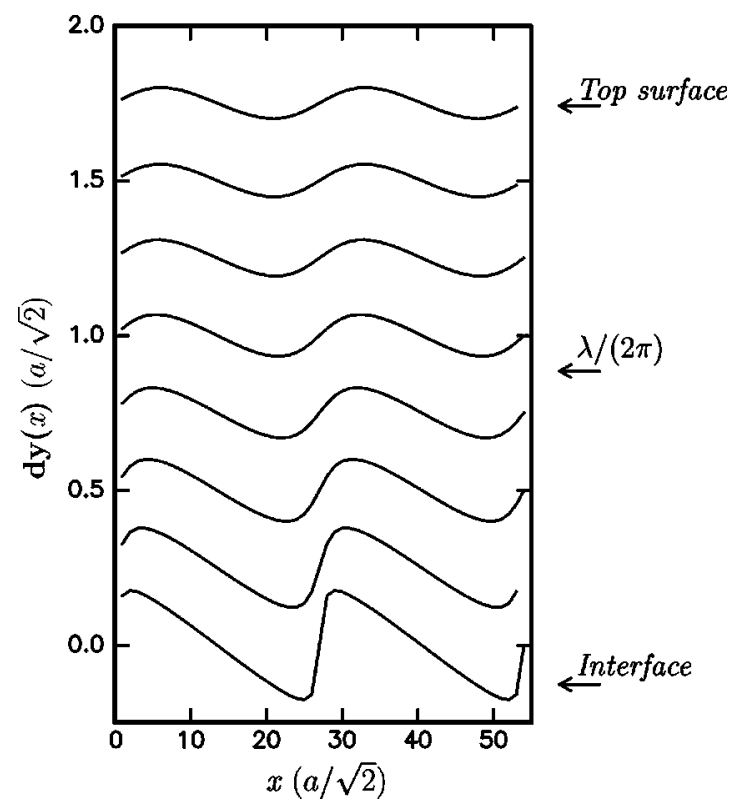

FIG. 3. Displacement wave in atomic layers parallel to the interface in the $4.3 \mathrm{~nm}$ upper crystal. The displacements $d y(x)$ in the $y$ direction are shown as a function of $x$, both in units of $a / \sqrt{2}$. The curves are for atomic layers, the lattice parameter is $a$, and for clarity they are shifted vertically 0.25 relative to each other. reconstruction as well as the nucleation properties in thinfilm growth.

The practical application of thin bonded $\mathrm{Si}$ wafers will still depend on the fabrication of samples combining the properties described above with controlled properties of the top surface including purity and roughness. An important next step will be to study such systems with standard ultrahigh-vacuum surface analysis techniques including scanning tunneling microscopy, and to use bonded wafers as templates for the epitaxial growth of ordered arrays of nanoclusters.

The authors acknowledge the help from the staff at HASYLAB beamline BW2. The work was supported by Dansync, the Danish National Material Research Program, the IHP program of the European Commission (HPRI-CT1999-0040), and BMBF Project No. 05 KS1GUC/3.

${ }^{1}$ M. Nielsen, R. Feidenhans'1, P. B. Howes, J. Vedde, K. Rasmussen, M. Benamara, and F. Grey, Surf. Sci. 442, L989 (1999).

${ }^{2}$ A. Bourret, Surf. Sci. 432, 37 (1999).

${ }^{3}$ A. E. Romanov, P. M. Petroff, and J. S. Speck, Appl. Phys. Lett. 74, 2280 (1999).

${ }^{4}$ F. Fournel, H. Moriceau, N. Magnea, J. Eymery, J. L. Rouvière, K. Rousseau, and B. Aspar, Mater. Sci. Eng., B 73, 42 (2000).

${ }^{5}$ F. Fournel, H. Moriceau, N. Magnea, J. Eymery, D. Buttard, J. L. Rouvière, K. Rousseau, and B. Aspar, Thin Solid Films 380, 10 (2000).

${ }^{6}$ Q.-Y. Tong and U. Gösele, Semiconductor Wafer Bonding: Science and Technology (Wiley, New York, 1999).

${ }^{7}$ F. E. Ejeckam, Y. H. Lo, S. Subramanian, H. Q. Hou, and B. E. Hammons, Appl. Phys. Lett. 70, 1685 (1997).

${ }^{8}$ R. A. Wind, M. J. Murtagh, F. Mei, Y. Wang, M. A. Hines, and S. L. Sass, Appl. Phys. Lett. 78, 2205 (2001).

${ }^{9}$ S. Weichel, F. Grey, K. Rasmussen, M. Nielsen, R. Feidenhans'1, P. B.Howes, and J. Vedde, Appl. Phys. Lett. 76, 70 (2000).

${ }^{10}$ J. P. Hirth and J. Lothe, Theory of Dislocations (Pergamon, New York, 1982).

${ }^{11}$ J. P. Hirth and B. Carnahan, Acta Metall. Mater. 40, 1237 (1992).

${ }^{12}$ P. B. Howes, M. Benamara, F. Grey, R. Feidenhans'1, M. Nielsen, F. B. Rasmussen, and J. Baker, Physica B 248, 74 (1998).

${ }^{13}$ L. D. Landau and E. M. Lifshitz, Theory of Elasticity (Pergamon, New York, 1959). 